\title{
Non Linear Modeling of Mixed Ionic Electronic Conductors
}

\author{
F. Ciucci, D.G. Goodwin \\ Division of Engineering and Applied Sciences, California Institute of Technology, \\ Pasadena, California, 91125, USA
}

\begin{abstract}
A nonlinear model for the study of mixed ionic electronic conductors (MIEC) is presented in this paper. The model is time dependent and takes into account electrical carriers motion, the electrical behavior of the MIEC-metal interface and the kinetics of the chemistry occurring at the MIEC surface. By applying a small potential input, complex impedances are computed.
\end{abstract}

\section{Introduction}

Mixed ionic electronic conductors are currently of great interest for SOFC applications. For example, ceria-containing anodes can be operated directly on hydrocarbons without coking, and in addition can be used at lower temperatures than $\mathrm{Ni}$ /YSZ (see Trovarelli (1)). In order to design, optimize, and characterize MIEC electrodes, it is very useful to have models to aid in interpreting experimental results. In this work, we present a nonlinear, time-dependent model for the study of MIECs. This model allows us to compute in time and space species concentrations, electric potential and currents. Poisson's equation is solved for the electric potential, coupled to nonlinear drift diffusion equations (DDE) for the relevant species in the bulk. Due to the high doping levels in MIECs, the DDE take into account interaction effects among defects. We also include the kinetics of reactions occurring at the MIEC surface and the triple phase boundaries. Since the model is formulated in the time domain and does not make smallsignal approximations, it is suitable to use to study nonlinear electrical excitation. As an example, linear impedance spectra are computed and illustrated.

\section{System Modeled}

The system we model is a MIEC slab placed between two metal electrodes (Figure 1). The MIEC is immersed in an oxidizing atmosphere consisting of $\mathrm{O}_{2}$ and a noble gas. The system is subjected to a constant total pressure, homogeneous temperature and variable partial pressure of $\mathrm{O}_{2}\left(\tilde{p}_{O_{2}}\right)$. We also assume that the MIEC is highly doped and the electric charge distribution of the dopant is negative. We further suppose the MIEC conducts electrons and oxygen ions or conversely vacancies.

The system under scrutiny is attached to an instrument that can measure the potential difference between the two electrodes and the current flowing within it. It is assumed that this instrument does not disturb the system under study and its dynamics is very "fast".

\section{Drift Diffusion Equation.}

In order to determine the electrical properties of the MIEC, one needs to solve Poisson's equation for the electric field and the conservation equations for each the species present in the sample, see Riess (2). We can then write the problem as follows: 


$$
\begin{aligned}
& \operatorname{div}(\operatorname{grad} \phi)=-\rho / \mathcal{E} \\
& \partial_{t} c_{e}+\operatorname{div} j_{e}=0 \\
& \partial_{t} c_{v}+\operatorname{div} j_{v}=0
\end{aligned}
$$

where $\phi$ is electric potential, $\rho$ the background charge, $\varepsilon$ the permittivity of the medium; $c_{\mathrm{e}}$ and $c_{\mathrm{v}}$ are the electron and vacancy density (number of electrons per unit volume) respectively. With $j_{\mathrm{e}}$ and $j_{\mathrm{v}}$ we indicate the fluxes of electrons and vacancies respectively.

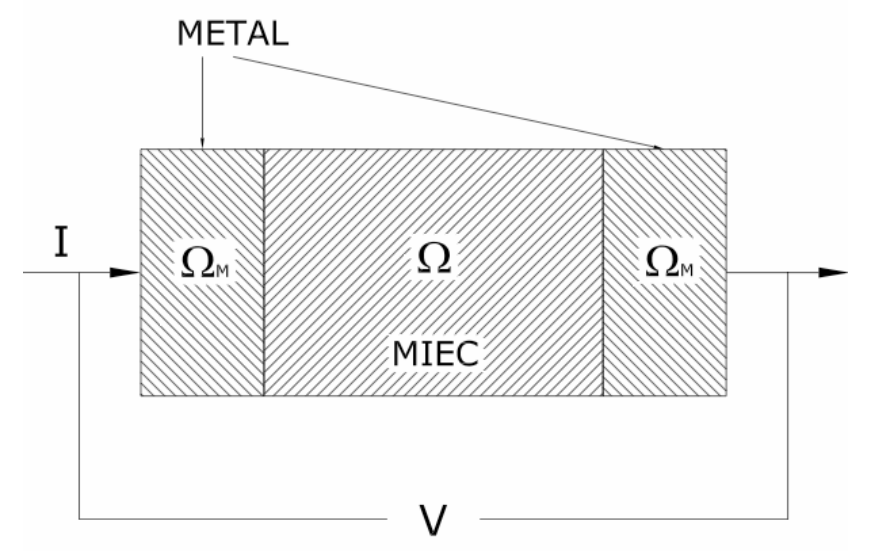

Figure 1. Schematic depiction of the MIEC assembly

We assume that the fluxes $j_{\mathrm{e}}$ and $j_{\mathrm{v}}$ are proportional to the gradients of electrochemical potential. For a generic species $X$ we can write:

$$
j_{X}=-\frac{c_{X} D_{X}}{k_{b} T} \operatorname{grad} \tilde{\mu}_{X}
$$

where $\tilde{\mu}_{X}$ is the electrochemical potential of the species $\mathrm{X}$. Since we assume oxidizing conditions and oxygen partial pressures greater than $10 \mathrm{~Pa}$; we also assume that $c_{\mathrm{e}}<<c_{\mathrm{V}}$ as indicated by Lai and Haile (3). Electroneutrality in the bulk together with equilibrium of the reaction:

$$
V_{O}^{\bullet \bullet}+\frac{1}{2} O_{2}+2 e^{\prime} \leftrightarrow O_{O}^{x}
$$

gives unperturbed/bulk concentrations of vacancies and electrons divided by the background concentration of dopants $B .^{1}$

We will consider the case in which electrons are dilute and follow a Boltzmann distribution, while vacancies are present in much greater number. It follows that site exclusion and interaction effects have to be taken into account. For lack of other sources Hendricks et al. approach is used (4) and we will express the electrochemical potentials in this way:

$$
\begin{aligned}
& \tilde{\mu}_{e}=\mu_{e}^{0}+k_{b} T \log \left(\frac{c_{e}}{c_{e}^{0}}\right)-e \phi \\
& \tilde{\mu}_{v}=\mu_{v}^{0}+k_{b} T \log \left(\frac{c_{v}}{c_{v}^{M A X}-c_{v}}\right)+2 e \phi
\end{aligned}
$$

\footnotetext{
${ }^{1}$ One needs to solve $p_{O_{2}}^{1 / 2} c_{e}^{2} c_{v}=K_{R}(T)$ (where the concentration of $O_{O}^{x}$ is unity) together with $c_{e}+B=2 c_{v}$. We take the value of $\mathrm{K}_{\mathrm{R}}$ from ref. 3 .
} 


\section{$\underline{\text { Adimensional Parameters and Equations }}$}

From the equations above a few adimensional parameters can be derived. First we can define:

$$
\begin{array}{ll}
U_{T}=\frac{k_{b} T}{e} & \lambda_{D}=\sqrt{\frac{\varepsilon U_{T}}{e B}} \\
\tau_{e}=\frac{\lambda_{D}^{2}}{D_{e}} & \tau_{v}=\frac{\lambda_{D}^{2}}{D_{e}} \quad \tau=\min \left(\tau_{e}, \tau_{v}\right)
\end{array}
$$

Then we will write the equations [1] in adimensional form using the transformations in [6].

$$
\begin{array}{ll}
\tilde{x}=x / \lambda_{D} & \tilde{t}=t / \tau \\
\tilde{\phi}=\phi / U_{T} & n=c_{e} /(\bar{n} B) \quad p=c_{v} /(\bar{p} B)
\end{array}
$$

This leads to the following expression of [1]:

$$
\begin{aligned}
& \Delta_{\tilde{x}} \tilde{\phi}=1+\bar{n} n-2 \bar{p} p \\
& \frac{\tau_{n}}{\tau} \partial_{\tilde{t}} n+\operatorname{div}_{\tilde{x}}\left(\operatorname{grad}_{\tilde{x}} \tilde{\phi}-\operatorname{grad}_{\tilde{x}} n\right)=0 \\
& \frac{\tau_{p}}{\tau} \partial_{\tilde{t}} p-\operatorname{div}_{\tilde{x}}\left(\frac{\alpha}{\alpha-\bar{p} p} \operatorname{grad}_{\tilde{x}} p+2 \operatorname{pgrad}_{\tilde{x}} \tilde{\phi}\right)=0
\end{aligned}
$$

\section{$\underline{\text { Initial and Boundary Conditions }}$}

Initial Conditions. In order to solve [7] we need appropriate boundary and initial conditions. Let us set that at $\mathrm{t}=0$ :

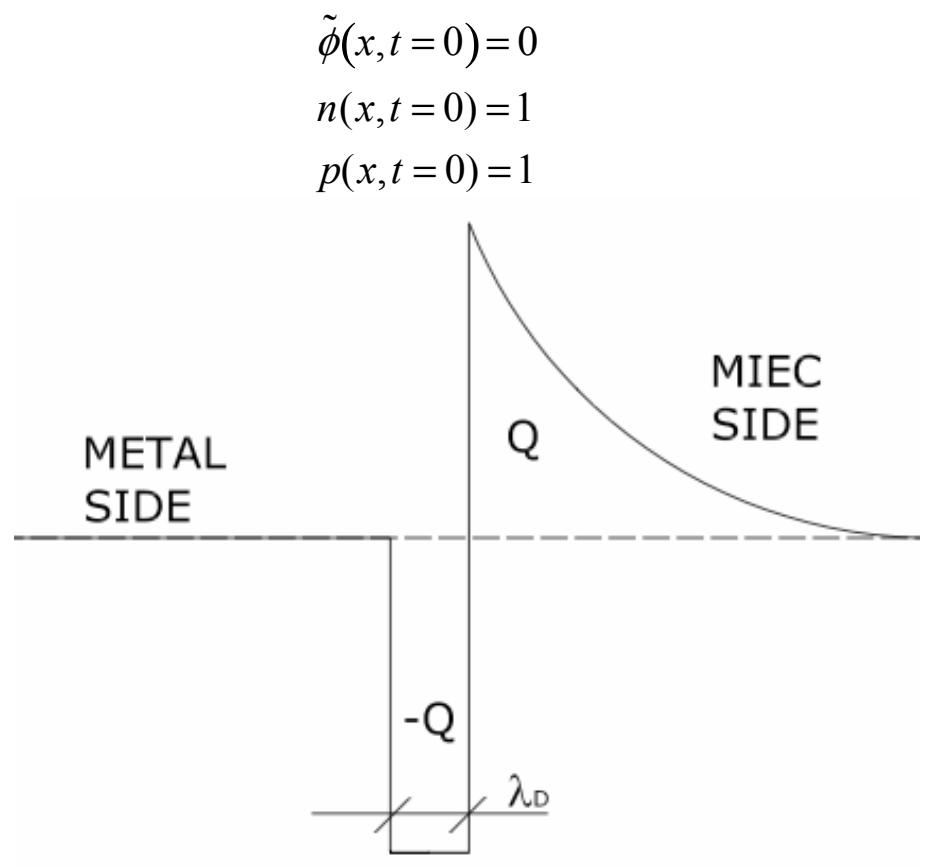

Figure 2. The Metal-MIEC interface. Depiction of the density of charge distribution near the MIEC-Metal Interface.

Potential. First, let us investigate the effect of a contact metal-MIEC. Note that if a net electric charge is present at the MIEC interface then a charge of opposite sign will be 
present at the metal surface. Suppose this charge is distributed uniformly over a length $\lambda_{S}$ and that the time response of the metal is infinitely fast. This implies that there will be an offset between the measured potential difference and the potential experienced by the MIEC at the interface; we will write:

$$
\tilde{\phi}(x, t)=\tilde{\phi}^{\text {electrode }}-\Delta \tilde{\phi}^{\text {surf }} \quad \text { s.t. } \quad \tilde{x} \in \partial \Omega \cap \partial \Omega_{M}
$$

where the drop in potential can be expressed by (see Figure 2.):

$$
\Delta \tilde{\phi}^{\text {surf }}=\frac{1}{2} \tilde{Q} \frac{\lambda_{S}^{2}}{\lambda_{D} \lambda_{D, m}}-\frac{\partial \tilde{\phi}}{\partial \tilde{x}} \frac{\lambda_{S}}{\lambda_{D}} \quad \text { s.t. } \tilde{x} \in \partial \Omega \cap \partial \Omega_{M}
$$

In the last expression, we introduced a few symbols:

$$
\lambda_{D, m}=\sqrt{\frac{\varepsilon_{m} U_{T}}{e B}} \quad \tilde{Q}=\int_{0}^{\tilde{L}_{m i d}}(2 \bar{p} p-\bar{n} n-1) d \tilde{x}
$$

and $\varepsilon_{\mathrm{m}}$ is the permittivity of the metal.

Flux of electrons. We note that since both MIEC and metal are electron-conducting electrons can migrate from the MIEC onto the metal and vice versa. This flux of electrons is often times linearized (Change-Jaffé boundary conditions see ref. 5 ) or not taken into account. Other times this flux boundary condition is substituted by an Ohmic boundary condition (electroneutrality is satisfied at the contact see Selberherr(6)). We shall take a different approach. Following the work of Bethe (7) and Sze (8) we will assume that the flux has the following form:

$$
\phi^{\prime M \leftrightarrow S}=k_{f, \text { surf }} B \bar{n} n e^{-\tilde{E}_{a c t}}\left(e^{\frac{1}{2} \Delta \phi^{s u f f}}-e^{-\frac{1}{2} \Delta \phi^{s u r f}}\right)
$$

where:

$$
k_{f, \text { surf }}=\sqrt{\frac{8 k_{b} T}{\pi m_{\text {polaron }}^{*}}}
$$

Kinetics of Reactions of MIEC. Following the work of Fleig (9), Fleig and Jamnik (10), Mebane and Liu (11) and experimental evidence by Lai (12) we will assume the following chemical kinetics for oxygen atoms (Figure 3.):

$$
\begin{aligned}
& O_{2}(g)+X(a d) \leftrightarrow O_{2}(a d) \\
& O_{2}(a d)+X(a d) \leftrightarrow O(a d) \\
& O(a d)+e^{\prime} \leftrightarrow O^{-}(a d) \\
& O^{-}(a d)+V_{O}^{\bullet} \leftrightarrow O_{O}^{x} \\
& V_{O}^{\bullet} \leftrightarrow V_{O}^{\bullet \bullet}+e^{\prime}
\end{aligned}
$$

where ( $g$ ) means gaseous species, (ad) means adsorbed species, $X$ indicates an unoccupied site on the surface and $\underline{V_{O}^{\bullet}}$ is a vacant oxygen site with charge +1 .

In [14] we will assume that I, II, IV and V are equilibrated, or conversely that [14.III] is the rate limiting step. We will also assume that $\left[V_{o}^{\bullet}\right]<<\left[e^{\prime}\right]<<\left[V_{O}^{\bullet \bullet}\right]$. Further assuming site exclusion of surface sites leads to write that: 


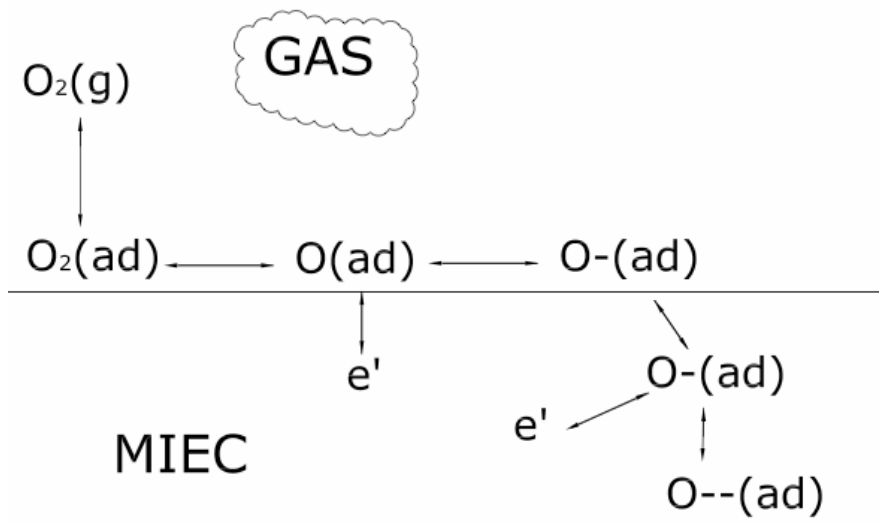

Figure 3. Illustration of the reaction kinetics occurring at the MIEC surface

$$
\begin{aligned}
& \mu_{O_{2}(g)}^{0}+k_{b} T \log \left(\frac{p_{O_{2}}}{p_{O_{2}}^{0}}\right)+\mu_{X}^{0}=\mu_{O_{2}(a d)}^{0}+k_{b} T \log \left(\frac{\vartheta_{O_{2}}}{\vartheta_{X}}\right) \\
& \mu_{O_{2}(a d)}^{0}+k_{b} T \log \left(\frac{\vartheta_{O_{2}}}{\vartheta_{X}}\right)+\mu_{X}^{0}=2 \mu_{O(a d)}^{0}+2 k_{b} T \log \left(\frac{\vartheta_{O}}{\vartheta_{X}}\right) \\
& \frac{d c_{e}}{d t}=k_{f} \vartheta_{O} c_{e} e^{(1-\alpha) \Delta \chi / U_{T}}-k_{r} \vartheta_{O^{-}} e^{-\alpha \Delta \chi / U_{T}} \\
& \mu_{O^{-}(a d)}^{0}+k_{b} T \log \left(\frac{\vartheta_{O}}{\vartheta_{X}}\right)-e \phi_{h}+\mu_{V_{o}^{*}}^{0}+k_{b} T \log \left(\frac{c_{V_{o}^{*}}}{c_{V_{o}^{*}}^{e q}}\right)+e \phi_{s u b}=\mu_{O_{O}^{x}}^{0} \\
& \mu_{V_{0}^{*}}^{0}+k_{b} T \log \left(\frac{c_{V_{o}^{*}}}{c_{V_{o}^{*}}^{e q}}\right)=\mu_{V_{o}^{*}}^{0}+k_{b} T \log \left(\frac{c_{v}}{c_{v}^{\max }-c_{v}}\right)+\mu_{e^{\prime}}^{0}+k_{b} T \log \left(\frac{c_{e}}{c_{e}^{0}}\right)
\end{aligned}
$$

where the $\underline{\vartheta}$ 's indicate site fractions (note that $\vartheta_{X}+\vartheta_{O^{-}}+\vartheta_{O}+\vartheta_{O_{2}}=1$ ). We will rewrite the [15]'s as follows:

$$
\begin{aligned}
& \vartheta_{O_{2}}=\alpha_{O_{2}} \vartheta_{X} \\
& \vartheta_{O}=\alpha_{O} \vartheta_{X} \\
& \frac{d c_{e}}{d t}=k_{f} \vartheta_{O}^{e q} c_{e}^{e q} e^{(1-\alpha) \Delta \chi^{e q} / U_{T}}\left[\frac{\vartheta_{O}}{\vartheta_{O}^{e q}} \frac{c_{e}}{c_{e}^{e q}} e^{(1-\alpha) \eta / U_{T}}-\frac{\vartheta_{O^{-}}}{\vartheta_{O^{-}}^{e q}} e^{-\alpha \eta / U_{T}}\right] \\
& \vartheta_{X}=\gamma \vartheta_{O^{-}} e^{\Delta \chi / U_{T}}
\end{aligned}
$$

where:

$$
\begin{aligned}
& \alpha_{O_{2}}=\frac{e^{\mu_{O_{2}(a d)}^{0}-\mu_{O_{2}(g)}^{0}-\mu_{X}^{0}}}{p_{O_{2}} / p_{O_{2}}^{0}}=\frac{\alpha}{\tilde{p}_{O_{2}}} \quad \alpha_{0}=\sqrt{\frac{\alpha_{O_{2}}}{e^{2 \mu_{O_{(a d)}}^{0}-\mu_{O_{2}(a d)}^{0}-\mu_{X}^{0}}}}=\sqrt{\frac{\alpha}{\beta \tilde{p}_{O_{2}}}} \\
& \gamma=\frac{c_{v}}{c_{v}^{\max }-c_{v}} \frac{c_{e}}{c_{e}^{0}} e^{\mu_{O_{O}^{X}-\mu_{v \sigma^{0}}^{0}-\mu_{e^{\prime}}^{0}}} \quad \frac{\Delta \chi}{U_{T}}=\frac{e^{2} \vartheta_{O^{-}} N_{0}}{C k_{b} T} \quad \eta=\Delta \chi-\Delta \chi^{e q}
\end{aligned}
$$

in which $N_{0}$ is the total number of surface sites available per unit of the free surface area. 
Finite Element Approximation. We will limit our study to a 1D model (Figure. 1) and in order to solve Eqs. [7] we will employ a traditional FEM method. We will omit mathematical subtleties and suppose the test and basis functions are continuous. We will further suppose the basis functions are quadratic and form a basis for the $P_{2, \mathrm{~K}}$ approximation of the unknowns ( $\mathrm{K}$ indicates the mesh and $P_{2}$ means that we are using polynomials of degree two). If $\psi_{i}$ is a basis element, then it is unitary at the i-th node and zero at all other nodes and its support is the mesh element which contains the i-th node. We can write any unknown function $a(\tilde{x})$ in a given mesh $K$ as $a(\tilde{x}) \cong P_{2, K}[a(\tilde{x})]=\sum_{i=1}^{N} a\left(\tilde{x}_{i}\right) \psi_{i}(\tilde{x})$. The initial system of PDE can then be approximated as a system of nonlinear algebraic-differential equations; in adimensional form it can be written as:

$$
\begin{aligned}
& \hat{K}_{i j} \tilde{\phi}_{j}+\hat{M}_{i j}\left(1+\bar{n} n_{j}-2 \bar{p} p_{j}\right)+b_{i}=0 \\
& \frac{\tau_{e}}{\tau} M_{i j} \dot{W}_{i}^{\prime}+\left(K_{i j} n_{i}-n_{i} \Lambda_{i j k} \tilde{\phi}_{k}\right)+\tau_{e} M_{i j} \dot{\delta}_{i}^{M I E C}+\frac{\tau_{e}}{\lambda_{D}} \dot{d}_{0}^{M \leftrightarrow S} \delta_{1 j}+\frac{\tau_{e}}{\lambda_{D}} \dot{\phi}_{L}^{M \leftrightarrow S} \delta_{N j}=0 \\
& \frac{\tau_{v}}{\tau} M_{i j} \dot{P}_{i}^{\prime}+\left(p_{i} \Lambda_{i j k} \tilde{\phi}_{k}+p_{i} \Lambda_{i j k} \tilde{\phi}_{k}\right)=0
\end{aligned}
$$

where:

$$
K_{i j}=\int_{0}^{L} \frac{d \psi_{i}}{d \tilde{x}} \frac{d \psi_{j}}{d \tilde{x}} d \tilde{x} ; \quad M_{i j}=\int_{0}^{L} \psi_{i} \psi_{j} d \tilde{x} ; \quad \Lambda_{i j k}=\int_{0}^{L} \psi_{i} \frac{d \psi_{j}}{d \tilde{x}} \frac{d \psi_{k}}{d \tilde{x}} d \tilde{x}
$$

and

$$
\hat{K}_{i j}=\left\{\begin{array}{ll}
K_{i j} & \text { if } i \neq 1, N \\
\delta_{i j} & \text { otherwise }
\end{array} ; \quad \hat{M}_{i j}=\left\{\begin{array}{cc}
M_{i j} & \text { if } i \neq 1, N \\
0 & \text { otherwise }
\end{array} ; \quad b=\left(\begin{array}{c}
-\phi_{a} / 2+\Delta \phi_{0}^{S} \\
0 \\
\vdots \\
0 \\
\phi_{a} / 2-\Delta \phi_{0}^{S}
\end{array}\right)\right.\right.
$$

\section{Results}

\section{Linear Simulations.}

We run the simulation outlined above with parameters written in Table 1 . The numerical procedure works as follows, first we reach a full nonlinear steady state condition, then compute the linearization of [19] and calculate the response of the system to an external harmonic excitation of the type $\phi_{a}=\phi_{0}+\varepsilon \phi_{0} e^{i \omega t}$ where $\varepsilon$ is "small". This gives a complex impedance. We report instead of the impedance the evaluation on the complex resistivity given as:

$$
\rho=\frac{1}{L \lambda_{D}} \frac{U_{T} \tau}{e B \lambda_{D}} \frac{\varepsilon \phi_{0} e^{i \omega t}}{\delta J}
$$

Two cases are reported. The first case (Figure 4) supposes that $\omega_{i}^{M I E C}=0$ at MIEC surface. While the second (Figure 5.) takes into account the kinetics of reactions occurring at the MIEC surface. Both plots indicate a decrease in $\rho$ 's modulus as the pressure decreases and indicate as well that $\rho$ increases if one adds MIEC kinetics according to the equations 
TABLE I. Parameters. (\# indicates number of particles or sites)

\begin{tabular}{cccccc}
\hline Parameter & Value & Parameter & Value & Parameter & Value \\
\hline$\lambda_{\mathrm{S}}$ & $1.25 \AA$ & $\tau_{\mathrm{e}}$ & $3.07 \mathrm{ps}$ & $\alpha$ & 0.01 \\
$\lambda_{\mathrm{D}}$ & $0.33 \AA$ & $\tau_{\mathrm{v}}$ & $6.25 \mathrm{ps}$ & $\beta$ & 50 \\
$\mathrm{~L}$ & 1000 & $\varepsilon / \varepsilon_{0}$ & 14 & $\gamma_{\text {bulk }}$ & 2.6 \\
$\mathrm{~T}$ & $600{ }^{\circ} \mathrm{C}$ & $\varepsilon_{\mathrm{m}} / \varepsilon_{0}$ & 1 & $\mathrm{C}$ & $100 \mu \mathrm{F} / \mathrm{m}^{2}$ \\
$\mathrm{~B}$ & $3.75 \mathrm{E} 27 \# / \mathrm{m}^{3}$ & $\mathrm{~m}^{*} / \mathrm{m}_{\text {electron }}$ & 10 & $\mathrm{k}_{\mathrm{f}}$ & 1.0 \\
$\mathrm{~N}_{0}$ & $1.35 \mathrm{E} 19 \# / \mathrm{m}^{3}$ & $\mathrm{C}_{\mathrm{v}, \max } / \mathrm{c}_{\mathrm{v}, \text { bulk }}$ & 7.25 & & \\
\hline
\end{tabular}

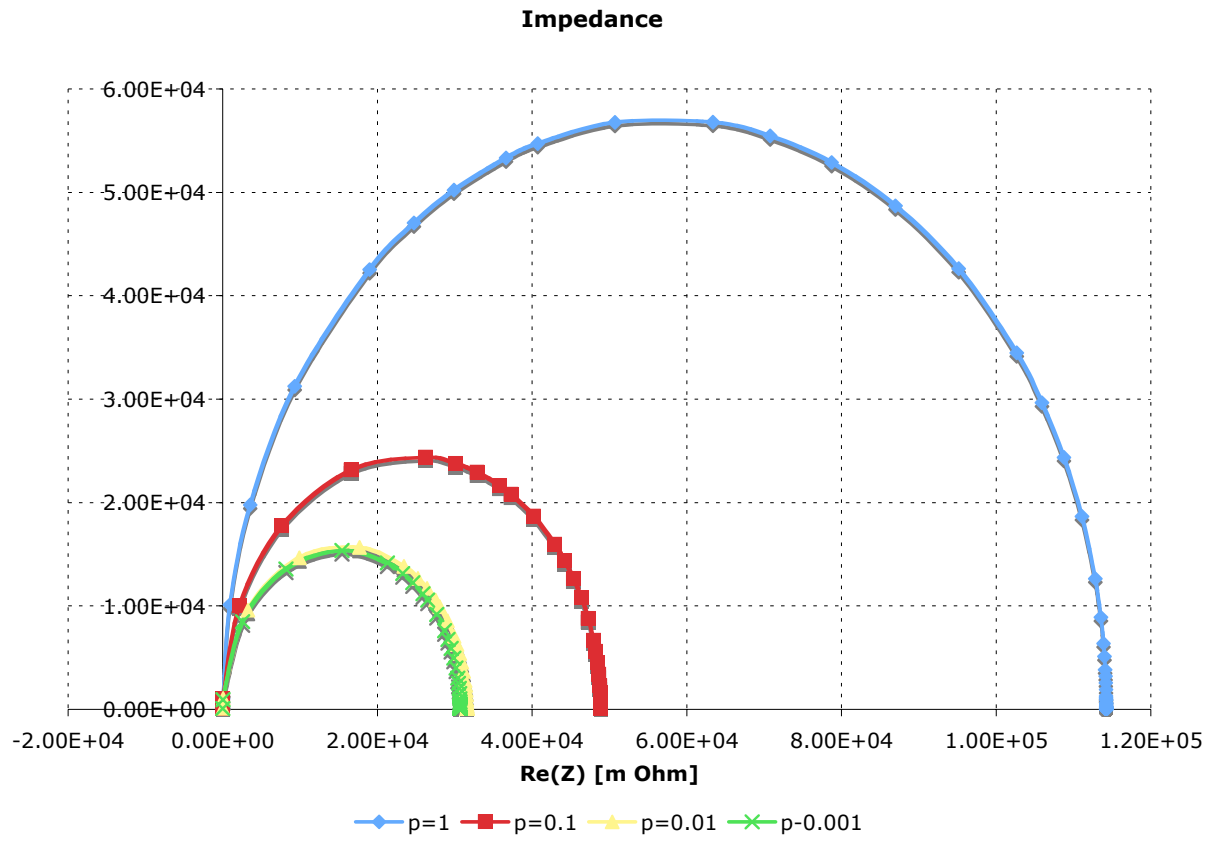

Figure 4. Impedance spectra - No MIEC Kinetics.

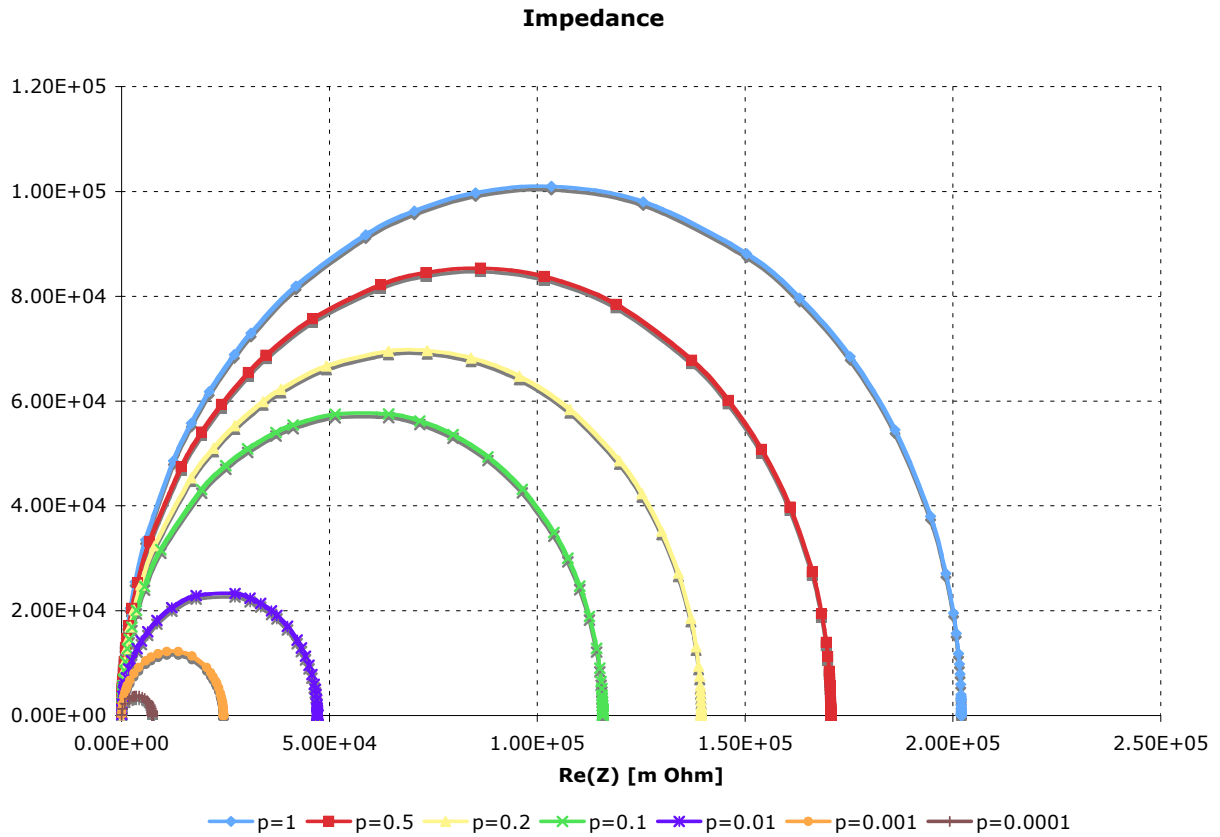

Figure 5. Impedance spectra including MIEC Kinetics. 
[15]. We also note that the spectra correctly indicate an ionic behavior of the material studied (12).

Work simulating nonlinear impedance spectra is in progress.

\section{Acknowledgments}

The authors gratefully acknowledge financial support for this work by Office of Naval Research under grant N00014-05-1-0712.

\section{References}

1. A. Trovarelli Ed., Catalysis by Ceria and Related Materials, World Scientific Publishing Company (2002)

2. I. Riess, Electrochemistry of Mixed Ionic-Electronic Conductors, p. 223, in CRC Handbook of Solid State Electrochemistry, P.J. Gellings and H.J.M. Bouwmeester, eds. CRC Press, Inc. (1997).

3. W. Lai and S.M. Haile, J. Am. Ceramic. Soc., 88 (11), 2979 (2005).

4. M.G.H.M. Hendricks, J.E. ten Elshov, and H.J.M. Bouwmeester et al. Solid State Ionics, 146 (3-4), 211 (2002).

5. H.G. Chang and G. Jaffé, J. Chem Phys., 20 (7), 1071-1077 (1952).

6. S. Selberherr, Analysis and Simulation of Semiconductor Devices, SpringerVelag, Wien (1984)

7. H. A. Bethe, M.I.T. Radiation Lab. Tech. Rep., 43-12 (1942).

8. S. M. Sze, Physics of Semiconductor Devices. New York: Wiley, 1981

9. Lai, PhD Thesis, California Institute of Technology (2006)

10. J. Fleig, Phys. Chem. Chem. Phys., 7 (9): 2027-2037 (2005)

11. J. Fleig and J.Jamnick J. Electrochem. Soc., 152, E138 (2005).

12. D.S. Mebane and M.L. Liu, J. Sol. State Electrochem., 10 (8), 575 (2006). 